\title{
Measurement of the Mechanical Deformation of Organ of Corti in a Model of Acute Endolymphatic Hydrops
}

\author{
Sang Heon Lee ${ }^{1}$ and Federico Kalinec ${ }^{2}$ \\ ${ }^{1}$ Department of Otolaryngology-Head and Neck Surgery, College of Medicine, Wonkwang University, Iksan, Korea \\ ${ }^{2}$ Laboratory of Auditory Cell Biology, Department of Head \& Neck Surgery, David Geffen School of Medicine at UCLA, LA, CA, USA
}

급성 내림프 수종 모델에서 코르티 기관의 기계적인 변형

이 상 헌 $^{1} \cdot$ Federico Kalinec ${ }^{2}$

원광대학교 의과대학 이비인후-두경부외과학교실,

청각세포생물학 연구소, 두경부외과학교실, 데이비드 게펜 의과대학, 캘리포니아 대학교 로스엔젤레스 ${ }^{2}$

\author{
Received September 8, 2015 \\ Revised November 26, 2015 \\ Accepted November 28, 2015 \\ Address for correspondence \\ Sang Heon Lee, MD, PhD \\ Department of Otolaryngology- \\ Head and Neck Surgery, \\ College of Medicine, \\ Wonkwang University, \\ 895 Muwang-ro, Iksan 54538, Korea \\ Tel $+82-63-859-1443$ \\ Fax $+82-63-841-6556$ \\ E-mail pericomo@wku.ac.kr
}

Background and Objectives Endolymphatic hydrops has been considered as an important histologic substrate of Meniere's disease. A permanent displacement of basilar membrane (BM) by increased endolymphatic pressure has been thought to be an explanation for hearing change. Direct observation of histological sections of temporal bones, however, suggested that stereocilia and tectorial membrane decoupling is more associated with pressure induced by mechanical deformation of the organ of Corti rather than with the displacement of BM.

Meterials and Method 26 cochleae from 13 female pigmented ginea pigs were harvested. One cochlea per each animal was injected with artificial perilymph. The other one was used as control. After fixation, followed by embedding and mid-modiolar sectionning, specimens were observed with a microscope. Morphometric parameters of each row and turn of the organ of Corti were measured and quantified.

Results The average area and height of the organ of Corti were significantly smaller in the apical turn of the experimental group $(p<0.05)$. The lengths of outer hair cell and Deiters cell in the apical turn were also significantly reduced in the experimental group $(p<0.05)$. The angle between the outer hair cell and Deiters cell was smaller in the apex and in the 3rd turn of the experimental group $(p<0.05)$.

Conclusion Results show that compression and deformation of the organ of Corti, especially in the apical turn, is a prominent feature in the acute endolymphatic hydrops model. We suggest that the deformation of organ of Corti is the primary cause of hydrops that induce the decoupling of tectorial membrane and stereocilia rather than the displacement of BM.

Korean J Otorhinolaryngol-Head Neck Surg 2016;59(2):110-9

Key Words Artificial endolymph injection - Endolymphatic hydrops · Guinea pigs · Histopathology $\cdot$ Meniere's disease.

\section{Introduction}

Endolymphatic hydrops (EH) represents a histopathologic finding in which the structures surrounding the scala media are distended by increased endolymphatic volume. It is acknowledged as important substrates of Meniere's disease
(MD). This finding was initially reported independently by Hallpike and Cairnes in England and Yamakawa in Japan in the temporal bones of patients with MD. Dispensability of structures which surround the scala media, such as Reissner's membrane, stria vascularis, the organ of Corti of the cochlea, saccule, utricle and semicircular canals of the vesti- 
bule, is known to be related to the mechanical compliance of each structures. ${ }^{1)}$ Variations in mechanical compliance of the structures may also explain the variations of hydrops of different regions in the same specimen. ${ }^{2)}$ Compliance of vestibular end organs are known to be higher than that of the cochlea with higher compliance in the saccule and relatively lower compliance in the semicircular canals. ${ }^{1)}$ Thus, compliance of the cochlea is thought to be the lowest in the inner ear (mechanically the strongest). Cochlea also showed spatial difference of the compliance that increases with distance from base to apex. ${ }^{3)}$ Among the fine structures of the inner ear, basilar membrane (BM) is considered to be the main mechanical component and has been studied in detail. BM is also considered as one of the important structural elements that dominate the stiffness of the scala media. ${ }^{4)}$ Displacement of the $\mathrm{BM}$ and resulted uncoupling of outer hair cell $(\mathrm{OHC})$ stereocilia from the tectorial membrane (TM) has been thought to be an explanation of hearing change. ${ }^{5)}$ However, estimated average compliance of the BM in the basal turn of the guinea pig is only $0.66 \mathrm{~nm} / \mathrm{Pa}{ }^{6)}$ Considering the degree of pressure difference which could be created by a few $\mu \mathrm{L}$ of artificial endolymph injection is only about $10 \mathrm{~Pa}$, the extent of $\mathrm{BM}$ displacement would be negligible with respect to the dimensions of the organ of Corti. Using given value for the volume compliance of the total $\mathrm{BM}(0.09 \mathrm{nl} / \mathrm{Pa})$, calculated average for the maximum displacement of the BM is $2 \mu \mathrm{m}$ if it is assumed that this volume displacement completely takes place at apical $3 \mathrm{~mm}$ of the BM. Such a displacement is still small in comparison with the dimensions of the organ of Corti. As a result, the organ of Corti, which is considered to be more elastic than BM, could be affected mechanically more by a condition in which the endolymphatic pressure is suddenly increased. Considering stiffness difference of the BM and the organ of Corti in the same space, this assumption could be more reasonable. ${ }^{4)}$

To evaluate the functional and structural consequences of acute EH, several types of animal models, for example, injections into the endolymphatic space, cerebrospinal fluid pressure manipulations, low-frequency sound, gel injection into the cochlea apex and cyclic adenosine monophosphate manipulations have been developed. ${ }^{7)}$ Among those models, the acute injection of an artificial endolymph into the endolymphatic space is considered as an apparently simple model of hydrops. ${ }^{8)}$ With this model, much useful electrophysiological and functional information about changes of endocochlear potential, summating potentials, compound action potential,
OAEs and perilymphatic potassium concentration after injection with various injection rates and amounts have been reported. ${ }^{9-11)}$ Most of the physiologic changes occurred transiently and recovered afterwards. Therefore, these changes were considered as results of increased pressure during injection. ${ }^{8)}$ Morphological and ultrastructural changes after induction of hydrops such as disarrangement of the stereocilia, interruption of cross-linkage system, displacement of nucleus, and degeneration of cell organelles also have been reported. $^{12,13)}$ Dunnebier and coworker ${ }^{13)}$ presented photos of cross sectional view of the organ of Corti after induction of two phase EH in guinea pig in which they showed morphological changes of the organ of Corti, however, they focused on the surface anatomy and the ultrastructural changes of cochlear cells, and made no comment about those changes. And also, all of them were changes after at least 1 month after induction of EH. The effect of increased endolymphatic pressure on the morphology of the organ of Corti immediately after acute injection into scala media is still elusive.

We hypothesized that sudden increase of endolymph would result in not only electrophysiological change but also micromechanical alteration of the inner ear, especially, microstructural changes of the organ of Corti other than BM, which would contribute to acute inner ear dysfunctions such as hearing loss, and possibly tinnitus. In order to prove this hypothesis, we designed ex vivo model of acute $\mathrm{EH}$ in which we could constantly maintain constant pressure in the endolymphatic system with artificial endolymph injection. Morphologic parameters such as area and height of the organ of Corti, height of the tunnel of Corti, length of OHCs, length of Deiters cells, width of reticular lamina, angles between OHCs and Deiters cells, and angles between reticular lamina and BM are measured.

\section{Meterials and Method}

For the experiments, a total of 26 cochleae from 13 young female pigmented guinea pigs, 4-8 week-old, were harvested under general anesthesia induced by intramuscular administration of ketamine/xylazine $(60 / 3.5 \mathrm{mg} / \mathrm{kg})$ following procedures approved by the institute's Institutional Animal Care and Use Committee. To promote viability and to reduce any possible damage of cochlear cells, temporal bones were immediately soaked in Leibovitz's L-15 Medium (Gibco ${ }^{\circledR}$; Life Technologies, Grand Island, NY, USA). One cochlea per animal was used as control and the other for the procedure. We built a system combining a surgical microscope (Zeiss OPMI 
Pico Technoscope Plus; Carl Zeiss Meditec, Dublin, CA, USA) with a goniometric base-platform where the dissected cochlea was placed. To measure intra-cochlear pressure and to inject artificial endolymph, a $0.2 \mathrm{~mm}$ hole was made in the lateral wall of the basal first turn of the cochlea using a $15 \pm 2 \mu \mathrm{m}$ tip diameter, $5-10 \mathrm{M} \Omega$ glass micropipette made by a P-97 micropipette puller system (Sutter Instrument, Novato, CA, USA). Micropipette was placed in a PESW-B17P electrode holder (Warner Instrument, Hamden, CT, USA) driven by a PatchMan electrode micromanipulator (Eppendorf, Hamburg, Germany). The electrode holder has an additional sealable port allowing for the insertion of the micropres-
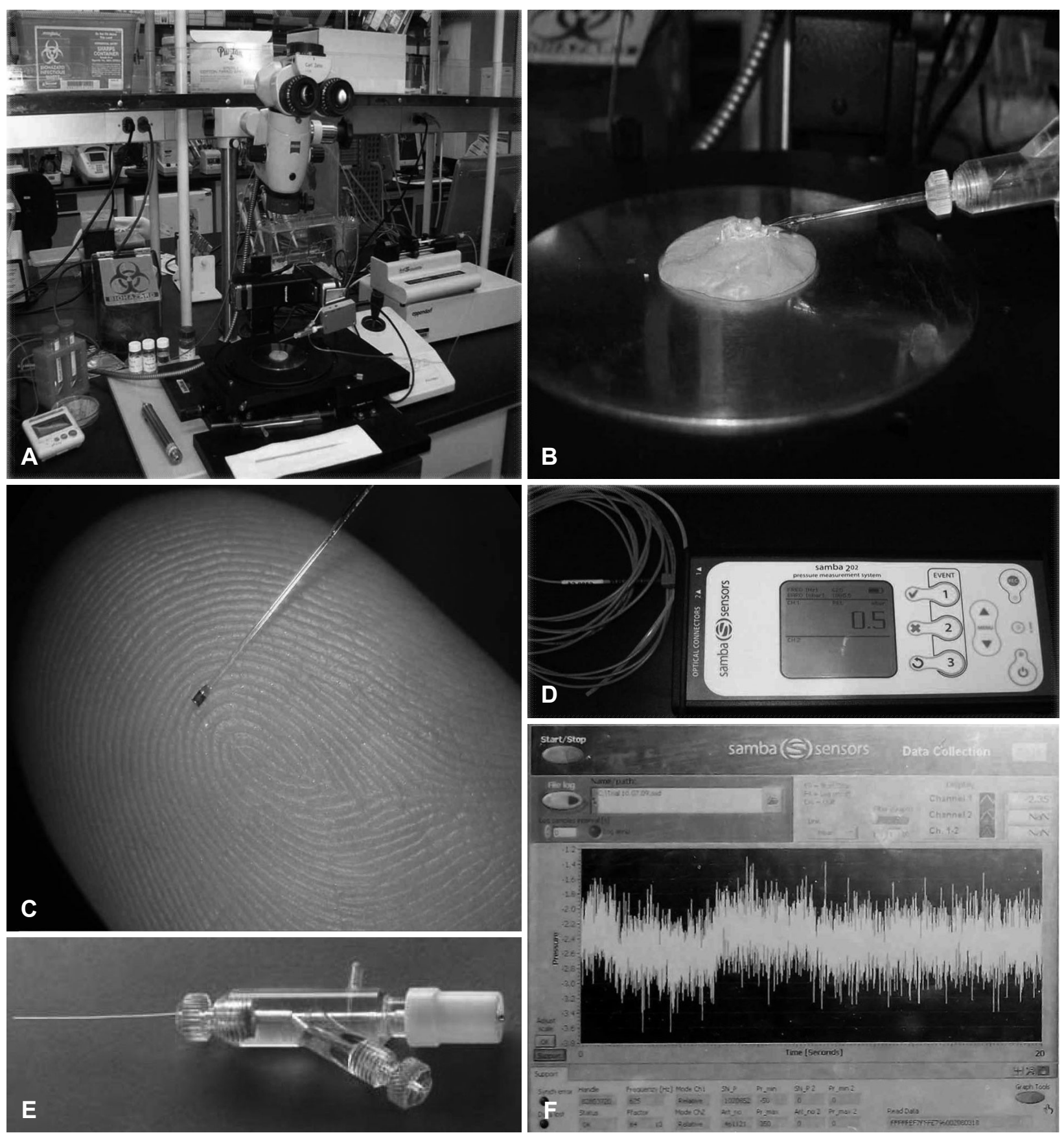

E

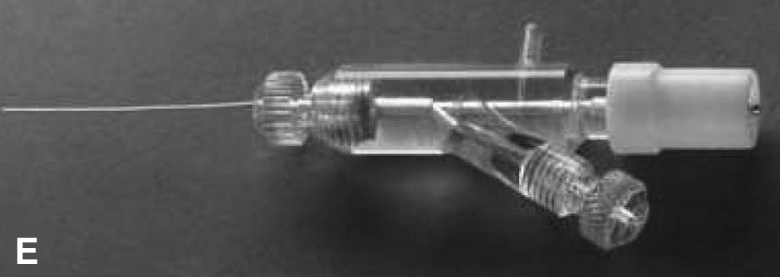

Fig. 1. Microinjection system (A and B) and pressure measurement system Samba MPM 100A-2-US (C-F). Experimental setup for microinjection system (A). Detail of the goniometric plate, with an open guinea pig bulla already in place and a glass micropipette in the pipette holder (B). Sensor 420 LP (0.25 mm-diameter; BIOPAC System, Inc., Goleta, CA, USA) on the tip of the optic fiber (C). The Samba 202 with the optic fiber pressure transducer on the left (D). The microneedle holder showing the upper port (angled at $\left.30^{\circ}\right)$ where the sensor is inserted, and the lower port where the pressure is applied (E). Computer screen showing a record (F). 
sure sensor inside the microneedle. Pressure measurements inside the scala media were performed with a computer-controlled MicroPressure Monitoring System Samba 202 (BIO PAC System, Inc.) (Fig. 1). This system allows to measure pressures in the range -5 to $35 \mathrm{KPa}$ with an error of $\pm 5 \mathrm{~Pa}$ at a sampling frequency of $40 \mathrm{KHz}$ with continuous recording. A KD Scientific syringe pump model 100 (KD Scientific, Holliston, MA, USA) was used to inject artificial endolymph (140 $\mathrm{mM} \mathrm{KCl}, 25 \mathrm{mM} \mathrm{KHCO}_{3}$ ) into the scala media through the opening made in the lateral wall of basal turn of the cochlea using 4 different rates $(0.005 \mathrm{~mL} / \mathrm{h}, 0.1 \mathrm{~mL} / \mathrm{h}, 0.025 \mathrm{~mL} / \mathrm{h}$, and $0.5 \mathrm{~mL} / \mathrm{h}$ ) for 3 minutes each until endolymphatic pressure reaches plateau (Fig. 2). After injection finished, cochleae were fixed immediately in 4\% paraformaldehyde. Fixed cochleae were decalcified with $0.1 \mathrm{M}$ Na-EDTA containing fixative for 2 weeks. The specimens were embedded in Celloidin ${ }^{\mathrm{TM}}$, sectioned along a mid-modiolar plane every $10 \mu \mathrm{m}$, stained with hematoxylin and eosin. All sections of specimens were viewed with a Zeiss Observer D1 Microscope (Carl Zeiss USA, NY, USA). Pictures were taken with software (AxioVision LE Version 4.6, Carl Zeiss MicroImaging GmbH, Germany) and a CCD color camera (AxioCam HR 13; Carl Zeiss, Göttingen, Germany), which was attached to one of the ports of the viewing head. With the protocol about microinjection of artificial endolymph written above, we could induce acute endolymphatic hydrops without rupture of the Reissner's membrane and confirmed histologically (Fig. 3A and B). Morphometric parameters of the organ of Corti were measured and quantified using image analysis software Image $\mathrm{J}$, version $1.48 \mathrm{~s}$ (available online at http://rsb.info.nih.gov/ij/index.html). These included area and height of the organ of Corti, height of tun-

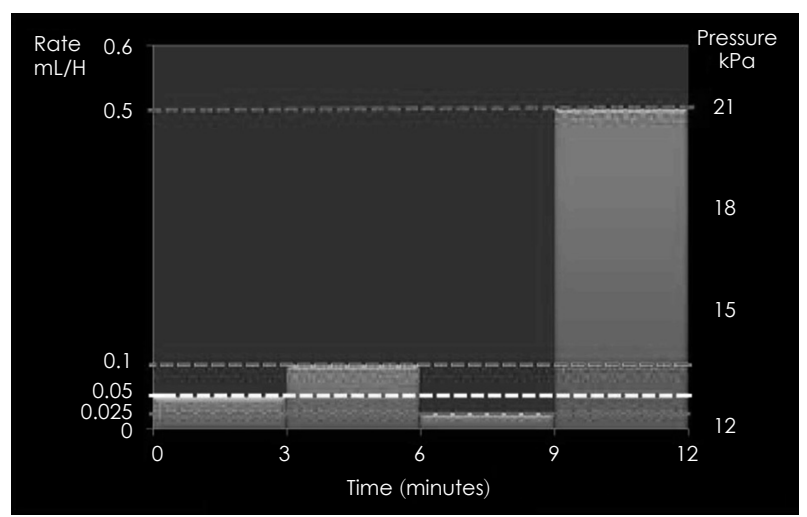

Fig. 2. Exprimental Protocol: before microinjection pressure was kept positive $(0.005 \mathrm{~mL} / \mathrm{h}-12.4 \mathrm{kPa})$ to prevent clogging of the microneedle. The data reported here corresponds to $\mathrm{AE}$ sequentially injected for a total of 12 minutes at $0.005 \mathrm{~mL} / \mathrm{h}\left(3^{\prime}\right), 0.1 \mathrm{~mL} / \mathrm{h}$ (3'), $0.025 \mathrm{~mL} / \mathrm{h}\left(3^{\prime}\right)$ and $0.5 \mathrm{~mL} / \mathrm{h}\left(3^{\prime}\right)$. AE: artificial endolymph. nel of Corti, length of OHCs and Deiters cells in each rows and turns, length of reticular lamina, angle between reticular lamina and BM and angles between OHCs and Deiters cell in each rows and turns (Fig. 3C and D). Area of the organ of Corti was measured by drawing an area contains a whole cellular structures of the organ of Corti from Hensen cells to inner hair cells. Height of tunnel of Corti was measured by drawing a perpendicular line between the upper middle point of the tunnel of Corti and the BM. For measurement of height of the organ of Corti, a vertical line between highest point of the organ of Corti and the BM was used. Length of $\mathrm{OHC}$ was determined as a distance between the reticular lamina in which stereocilia are located and the midpoint between the nucleus of $\mathrm{OHC}$ and Deiters cell of same row. Deiters cells have relatively narrow path of attachment in the BM. Length of Deiters cell was defined as a distance between attachment site of BM to the midpoint between nucleus of Deiters cell and $\mathrm{OHC}$ of same row. Reticular lamina is composed of apical surface of OHCs and phalangeal process of Deiters cells. Width of the reticular lamina was defined as upper straight portion of the organ of Corti from the junction between Hensen cell and $\mathrm{OHC}$ to the junction between $\mathrm{OHC}$ and outer pillar cell. Angles between the reticular lamina and the $\mathrm{BM}, \mathrm{OHCs}$ and Deiters cells of each rows and turns were measured, respectively. Statistical analysis was performed using Student t-test techniques (JMP 9 software; SAS Institute, Cary, NC, USA) and $p<0.05$ was regarded as the criterion for statistical significance.

\section{Results}

Average area of the organ of Corti of experimental group was significantly smaller than control group only in apical turn. Similarly, comparison of average height of the organ of Corti between control and experiment showed a tendency to become smaller toward the base. And only in apical turn, decrease of height of the organ of Corti was significant (Fig. 4) $(p<0.05)$. Average height of tunnel of Corti in experimental group was significantly smaller than control group $(p<0.05)$. In apical turn and third turn, heights were significantly decreased in experimental group (Fig. 5) $(p<0.05)$. Average length of OHCs in experimental group was significantly smaller than that of experimental group $(p<0.05)$. Length of 1st row $\mathrm{OHCs}$ in experimental group was significantly smaller than experimental group. In other rows, there were no significant differences. Also, length of OHC in apical turn of experimental group showed significant difference (Fig. 6) $(p<0.05)$. It 

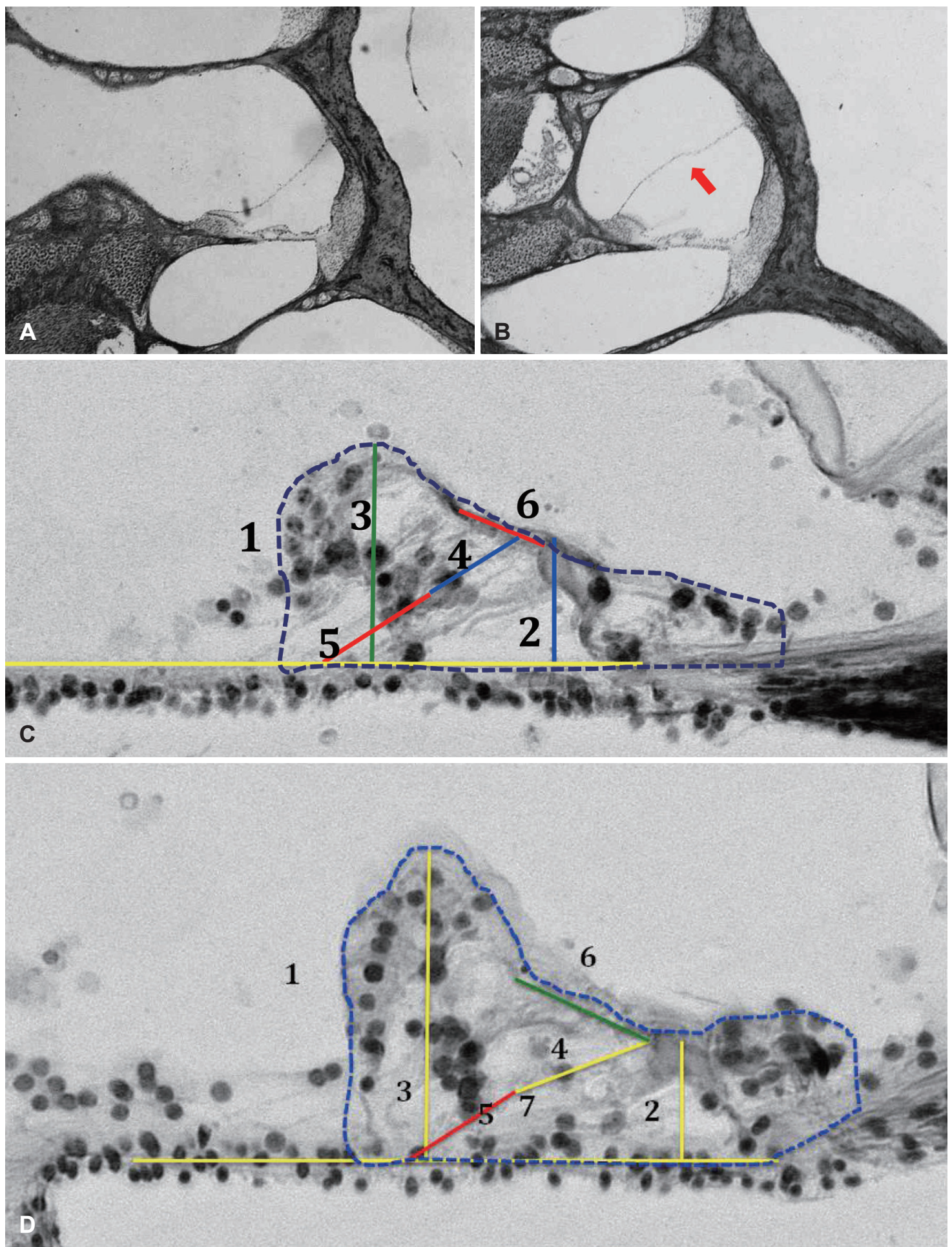

Fig. 3. Histology of cochlea (A: control, B: after injection of artificial endolymph) and morphologic parameters measured in the organ of Corti (C: control, D: experiment) Microinjection of artificial endolymph induces acute endolymphatic hydrops without rupture of the Reissner's membrane. Notes distension of Reissner's membrane (red arrow in B) and changes in the corresponding area of scala media and scala vestibule induced by artificial endolymph injection. 1: Area of organ of Corti (blue dotted line). 2: Height of tunnel of Corti (short vertical yellow line), 3: Height of organ of Corti (length between uppermost part of organ of Corti and basilar membrane, long vertical yellow line). 4: Length of OHC (yellow oblique line), 5: Length of Deiters cell (red oblique line). 6: Length of upper surface of 3 rows of OHCs (green oblique line). 7: Angle between OHC (4) and Dieters cell (5, not shown). 8: Angle of OHC [angle between 1st OHC (4) and basilar membrane (horizontal yellow line, not shown)]. 9: Angle of IHC (angle between IHC and basilar membrane, not shown). 10: Angle of reticular lamina [angle between reticular lamina (6) and basilar membrane (horizontal yellow line), not shown]. OHC: outer hair cell, IHC: inner hair cell. 

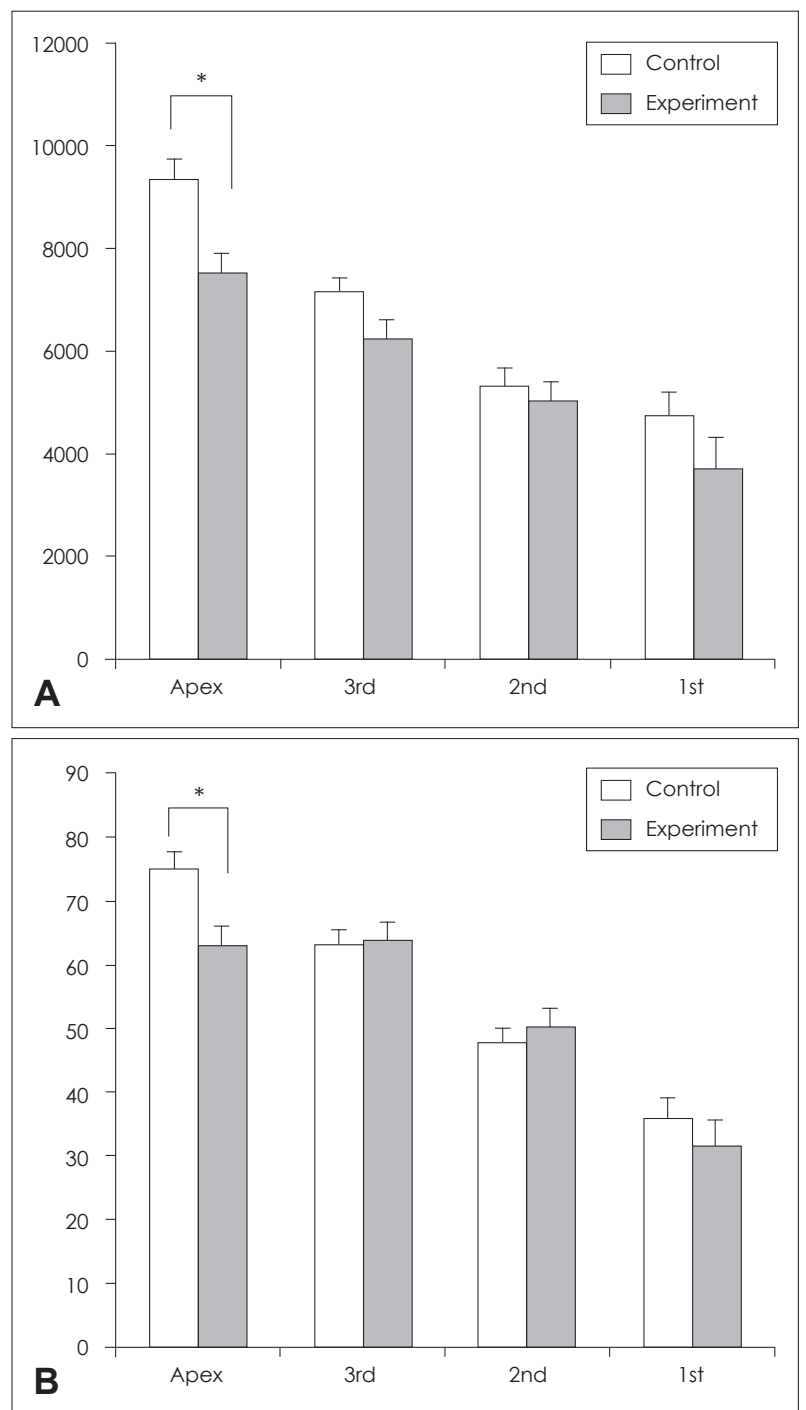

Fig. 4. Average area of organ of Corti of each turns of cochlea $\left(\mu \mathrm{m}^{2}\right)$. Area is significantly smaller only in apex (A). Height of organ of Corti. It showed a tendency to become smaller toward the base, however, only in apical turn, it showed a significant difference $\left({ }^{*} p<0.05\right)(\mathrm{B})$.

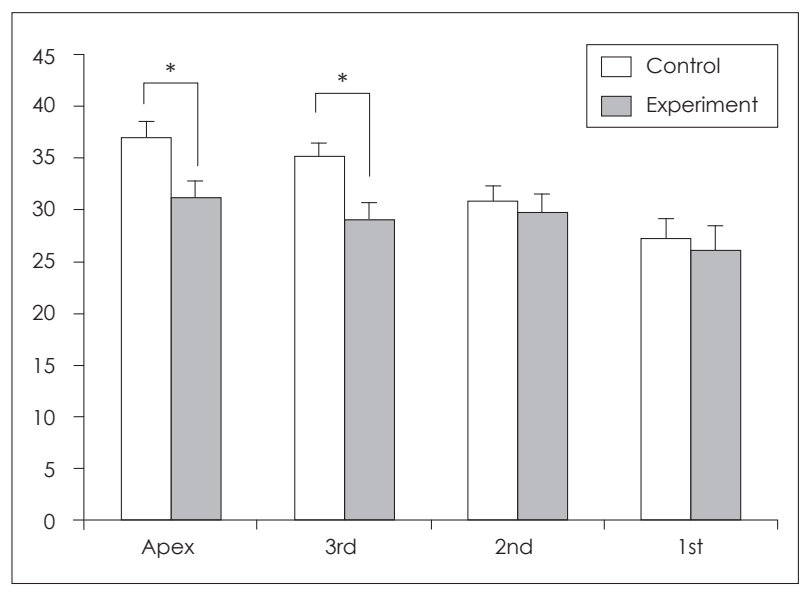

Fig. 5. Height of tunnel of Corti. * $p<0.05$. was smaller than that of control group. Average length of Deiters cell in experimental group is significantly smaller than control group $(p<0.05)$. In each rows, there showed significant differences of the length of Deiters cell between two groups. Significant differences were detected in apical turn and 3rd turn (Fig. 7) $(p<0.05)$. Also, length of Deiters cell of 2nd turn of experimental group was significantly longer than other turns $(p<0.05)$. For width of reticular lamina, there was no significant difference between average values of control and experimental group. However, width showed a tendency to become smaller toward base. There was no difference between 2nd turn and 3rd turn, but average width of apical turn is significantly wider and that of basal turn is significantly narrower (Fig. 8) $(p<0.05)$. Measurement of angles between reticular lamina and BM showed a tendency to become small-
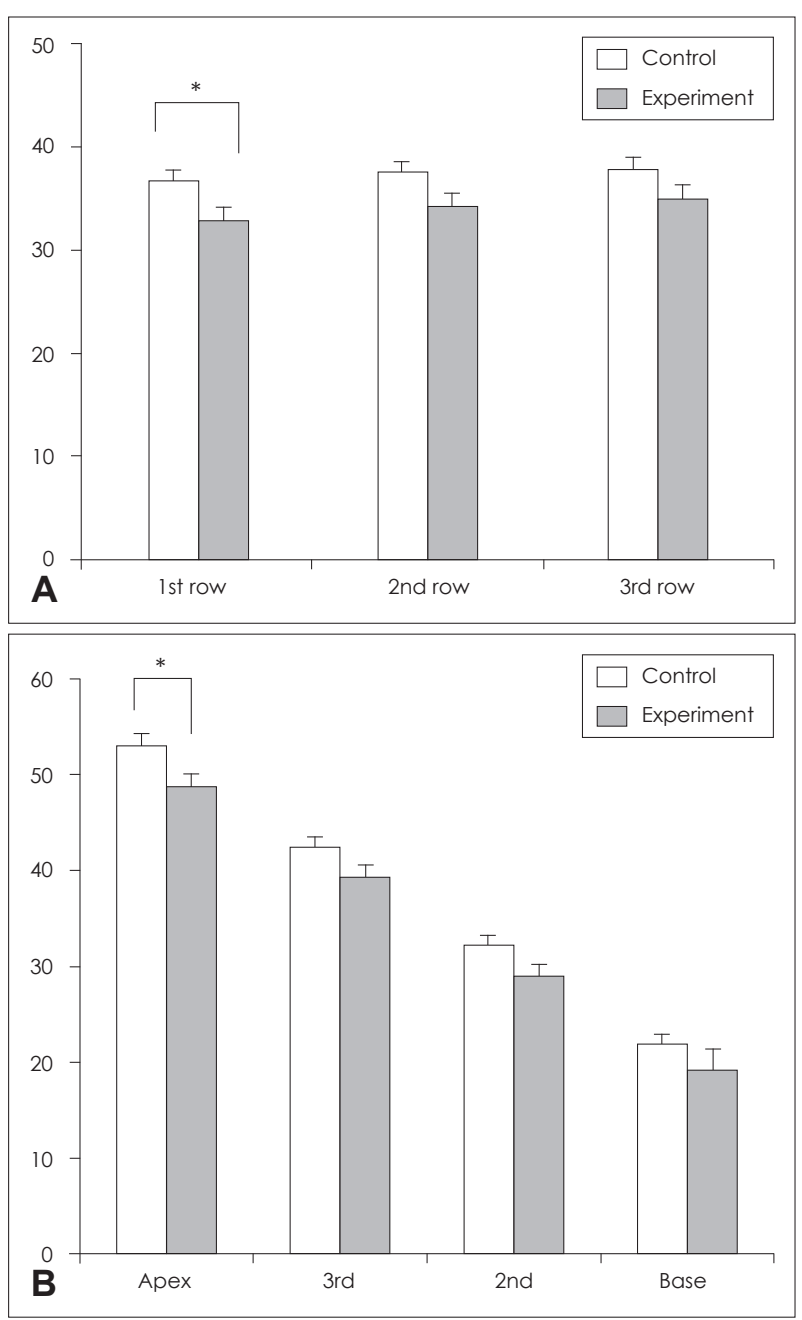

Fig. 6. Length of outer hair cells in each row. In 1st row, length was significantly reduced in experimental group $\left({ }^{*} p<0.05\right)(A)$. Length of outer hair cells in different cochlear turns. Only in apical turn, there is a significant difference between control and experiment $\left({ }^{*} p<0.05\right)(B)$. 

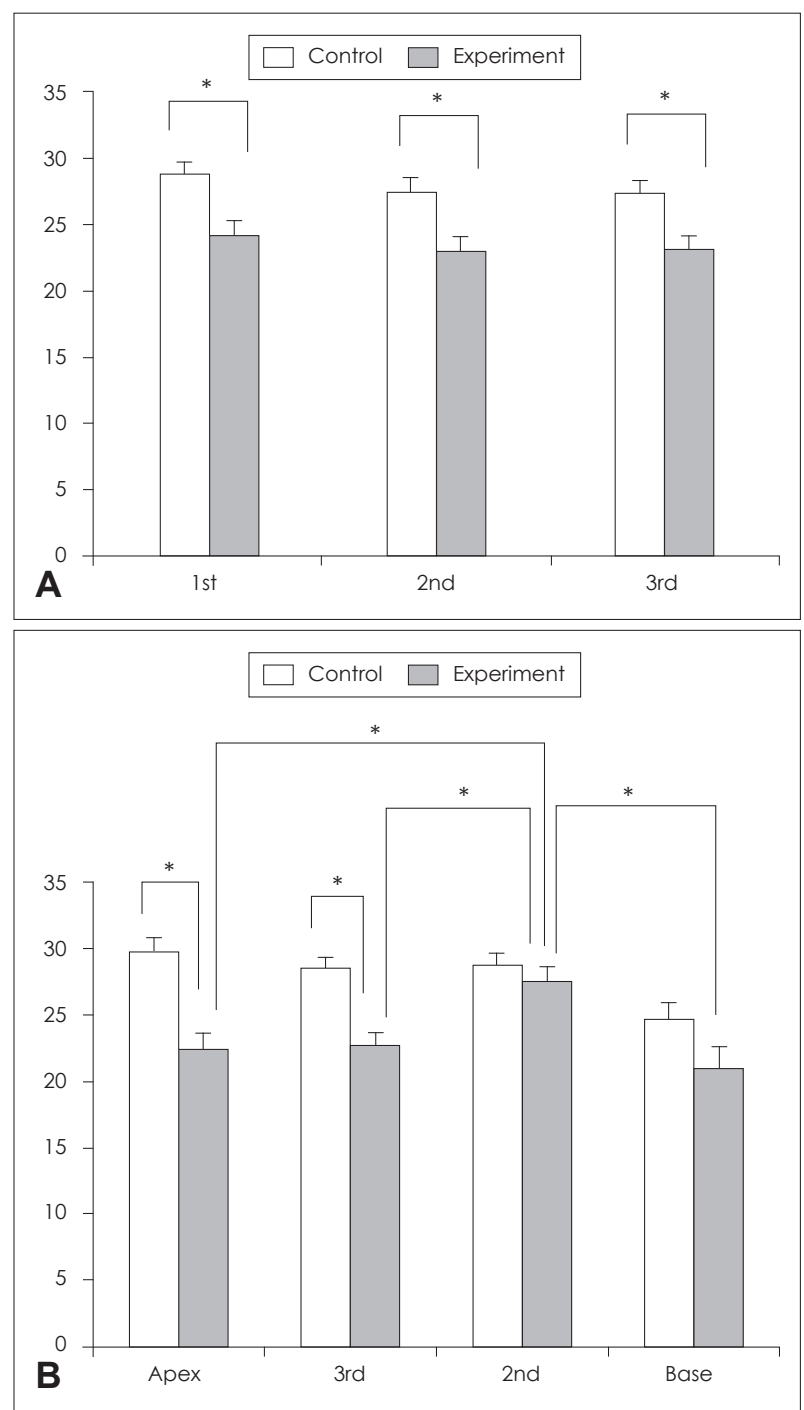

Fig. 7. Length of Deiters cell in each rows. There showed significant differences of the length of Deiters cell between two group $\left({ }^{*} p<0.05\right)(A)$. Length of Dieters cell in each turns. There were significant differences in apical turn and 3rd turn. Also, length of Deiters cell of 2 nd turn of experimental group was significantly longer than other turns $\left({ }^{*} p<0.05\right)(B)$.

er toward base, however, we couldn't find any significant changes between two groups, turns and rows. Angles between OHCs and Deiters cells of 1st row are the largest and it become smaller toward 3rd row. Its differences between rows were significant (Fig. 9) $(p<0.05)$. However, there was no significant difference between control and experiment. Angles of basal turn and 2nd turn are larger than those of 3rd turn and apex (Fig. 10) $(p<0.05)$. In apex and base, there were no significant difference between control and experiment. In 2nd turn, angles of experimental group were significantly larger than those of control. In 3rd turn, inversely, angles of experimental groups were significantly smaller $(p<0.05)$.

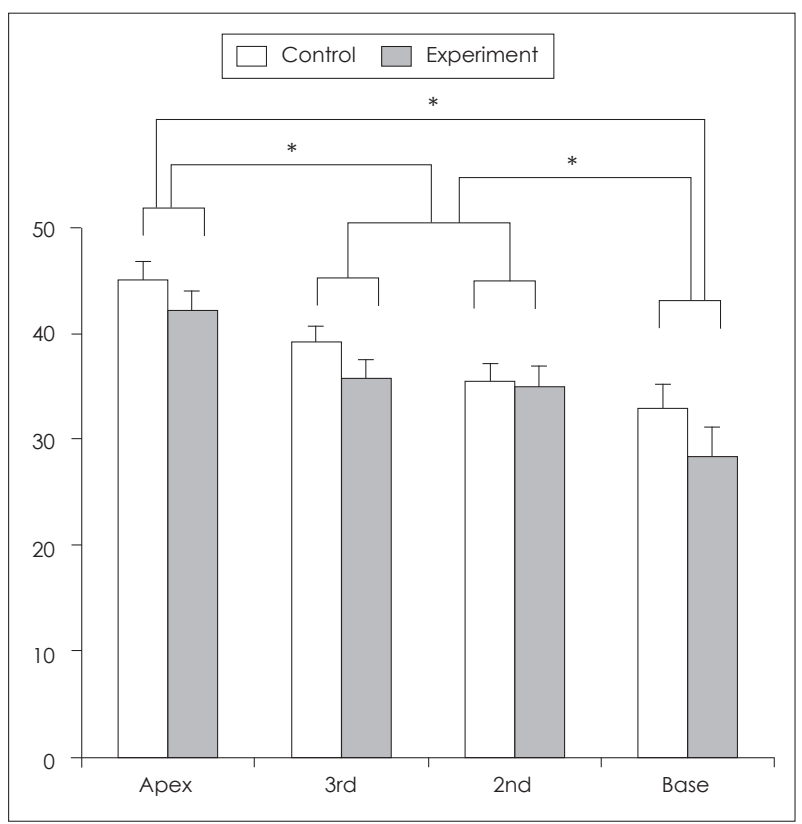

Fig. 8. Width of reticular lamina in each turns. There was no difference between 2nd turn and 3rd turn, but average width of apical turn is significantly wider and that of basal turn is significantly narrower $\left({ }^{*} p<0.05\right)$.

\section{Discussion}

EH is manifested by microscopic distension of the inner ear membrane. It may be clinically evident by disabling attacks of vertigo accompanied by low-tone hearing loss and tinnitus or merely by a progressive low-frequency hearing loss. Microscopic evidence of distension of the inner ear membranes (Reissner's and saccular) was first associated with clinical symptoms independently by Hallpike and Cairns and Yamakawa in patient with recurrent vertigo accompanied by fluctuating low-tone hearing loss and roaring tinnitus. The distortion of the membranes is now known as $\mathrm{EH}$ and the symptom complex as MD. EH may occur without the symptom complex of MD and be manifested solely by a low-frequency hearing loss that may or may not fluctuate, or the symptoms may occur without hydrops. Therefore, the term Meniere's syndrome should probably be used until hydrops can be demonstrated histologically.

Many theories have been proposed linking EH with the symptoms of MD. For example, Schuknecht proposed that an overdistension of the endolymphatic compartment could lead to leakages, mixing the high $\mathrm{K}^{+}$endolymph with the perilymph. This " $\mathrm{K}^{+}$poisoning" would be responsible for acute Meniere's attack, and resealing of the rupture would leads to their resolution. Yoon, et al. ${ }^{14)}$ extended this idea by proposing that overlay stretch of the Reissner's membrane could lead to 

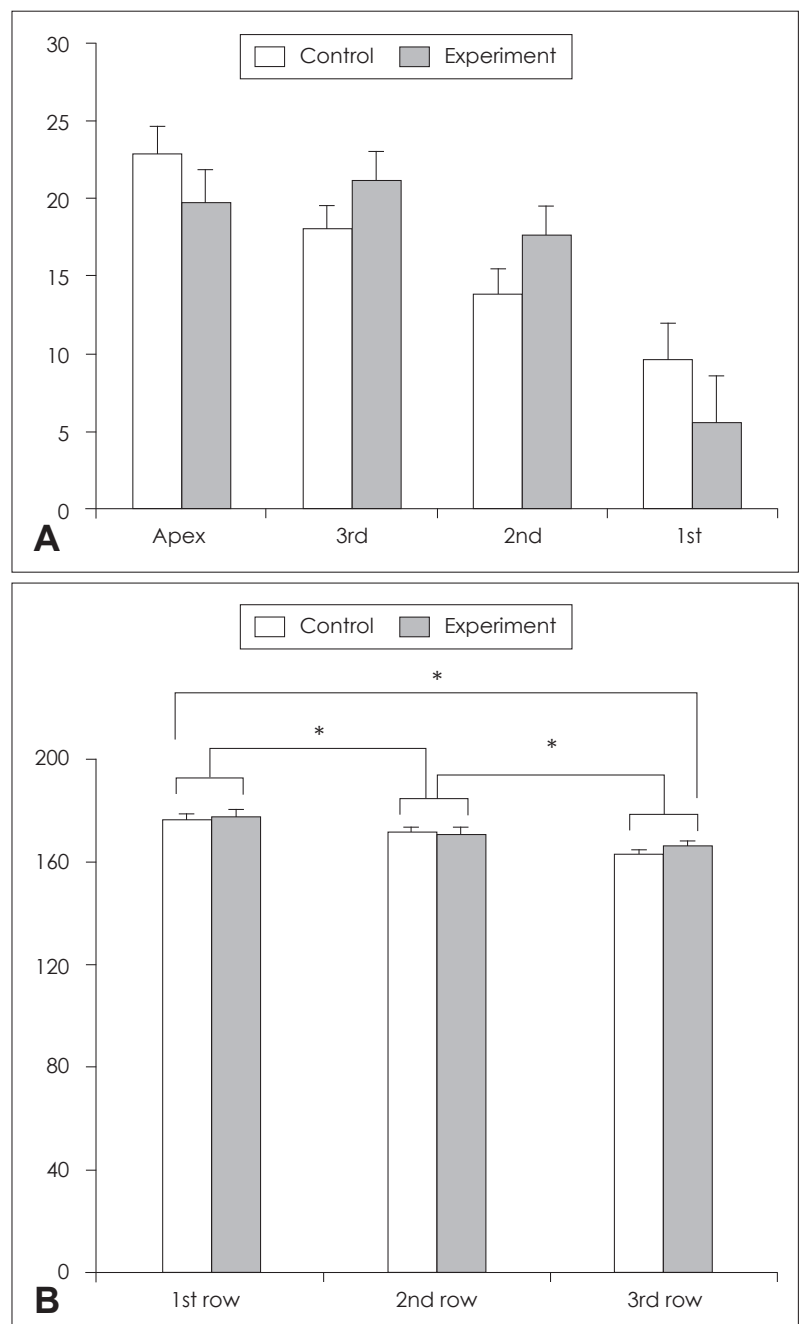

Fig. 9. Angle between reticular lamina and basilar membrane. There showed no significant differences between two groups (A). Angle between outer hair cells and Deiters cells. There showed no difference between control and experiment, however, angles become smaller toward 3rd row. Angles of 3rd rows are significantly smaller than other rows and angles of 2 nd row are smaller than 1 st row (B). * $p<0.05$.

improper ion transport and endolymph leakage without an actual rupture of the membrane. Tonndorf, ${ }^{5)}$ on the other hand, suggested that EH induce mechanical changes in the cochlea, and that hearing loss could be a direct consequence of alterations in the traveling wave. In addition, he proposed that the static shear between the TM and the organ of Corti caused by the displacement of the BM, may partially decouple the $\mathrm{OHCs}$ from the TM, an event that would explain the tinnitus, recruitment and decrease in speech intelligibility associated with EH. Experimental studies by Rydmarker and Horner ${ }^{15)}$ showed elevation of the TM and diminished imprints of the stereocilia on the undersurface of the TM, shears the corresponding stereocilia were intact, supporting Tonndorf's idea.

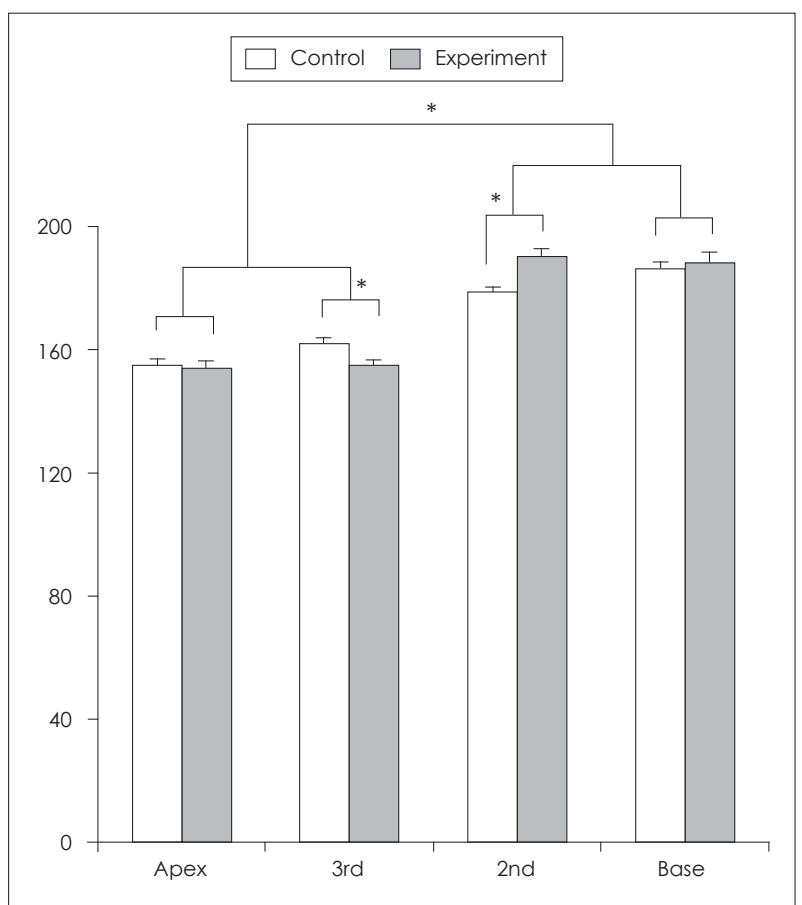

Fig. 10. Angle between OHCs and DCs. In apex and base, there was no difference between control and experiment. In 2nd turn, angles of experimental group were significantly larger than that of control. In 3rd turn, inversely, angles of experimental groups were significantly smaller $\left({ }^{*} p<0.05\right)$. Angles of basal turn and 2 nd turn are larger than those of 3rd turn and apex $\left({ }^{*} p<0.05\right)$. OHCs: outer hair cells, DCs: deiters cells.

A synthesis of Schucknecht and Tonndorf ${ }^{5)}$ theories was proposed by Zenner and coworkers, ${ }^{16)}$ involving motor disturbances in the hair cells and transitory endolymph leakage induced by $\mathrm{EH}$ in the pathophysiology of MD. These authors suggested that two basic mechanisms could play a key role in the pathogenesis of MD: 1) Chronic EH would generate an overproduction that alters the cochlear amplifier by decoupling the OHCs from the TM. This mechanism would contribute to chronic sensorineural hearing loss (SNHL) and tinnitus, but not to the acute attack. 2) Transitory endolymph leakages, resulting in acute $\mathrm{K}^{+}$intoxication of the perilymph, would result in irreversible damage to vestibular and cochlear hair cells. Vestibular damage would be compensated by the central nervous system, but SNHL should be permanent. For Zenner, et al., ${ }^{16)}$ both mechanism dramatically influence the function of vestibular, inner hair cells and OHCs, and the resulting disturbance of major hair cells functions-mechanoelectrical transduction (MET) and cochlear amplification-would be the origin of the typical symptoms of MD. This possible leakage of potassium was proved in the experiment of Kakigi and his co-workers ${ }^{9)}$ using animal model of acute EH. They observed perilymphatic potassium change after injection of artificial 
endolymph (KCL $140 \mathrm{mM}, \mathrm{KHCO}_{3} 25 \mathrm{mM}$ ) into the scala media of 2 nd turn of cochlea in guinea pig with rate up to 500 $\mathrm{nL} / \mathrm{min}$ for $10 \mathrm{~min}$. For injections of $\leq 3 \mu \mathrm{L}$, changes were small, but with larger volume injection, perilymphatic potassium concentrations were substantially increased. With these results, they suggested that substantial hydrops could result in increased perilymphatic potassium concentration, which could contribute to auditory and vestibular dysfunction.

The theory of Zenner and co-workers ${ }^{16)}$ introduced the concepts of $\mathrm{OHC}$ motor function and cochlear amplification in the MD. It suggests that acute $\mathrm{K}^{+}$intoxication would inhibit MET and cochlear amplification by inducing a sustained but reversible $\mathrm{OHC}$ depolarization, which in turn would translate into $\mathrm{OHC}$ shortening and the consequent deformation of the organ of Corti such as decrease in the distance between the reticular lamina $(\mathrm{RL})$ and the $\mathrm{BM}$. The unwanted $\mathrm{OHC}$ contraction, the possible TM-hair cell decoupling and the inhibition of OHC's MET would inhibit the cochlear amplifier. ${ }^{17)}$ The operating conditions of the OHCs would be drastically disturbed (loss of TM control, loss of MET control, change of operating points), so that the physiological amplification of the traveling wave near threshold would be out of control. These changes would explain the loss of OAEs, of frequency selectivity (speech discrimination, diplacusis), the increase in the auditory threshold (hearing loss) as well as tinnitus and recruitment associated with MD. Curiously these authors provided an extensive discussion of the possible effects of stereocilia-TM decoupling, but did not advance beyond Tonndorf ${ }^{5)}$ in explaining the mechanisms that cause this decoupling as a "basilar membrane displacement".

The results that the area and height of the organ of Corti were significantly reduced in the apical turn are presumed to represent that there would be some differences between the compliances of each turns of organ of Corti. It is also presumed that the organ of Corti in apical turn is sensitive to the change of endolymphatic pressure. Significant change of height of tunnel of Corti, length of 1st row $\mathrm{OHC}$ in apex and length of Deiters cell in apex and 3rd turn showed in present experiment is assumed to be that these 3 structures may play an important role in pressure compensation of organ of Corti. Also, these results are assumed to mean that these 3 parameters would have some important role in determining the compliance of organ of Corti. The result that there were no changes in width of reticular lamina and angle between reticular lamina and BM is thought to be partly due to evenly spread endolymphatic pressure upon the reticular lamina which was proved to have greater strength and resistance than other structures. However, because of the proximity and relatively weakness, adjacent structures such as tunnel of Corti, Hensen cells were thought to be more influenced by increased endolymphatic pressure and changed significantly in parameters such as height of organ of Corti and height of tunnel of Corti.

The results of the present study are consistent with the theory of Zenner and co-workers, ${ }^{17)}$ but provide new evidence suggesting that compression and deformation of the organ of Corti especially in the apical turn-rather than displacement of the BM-would be the primary cause of the hydrops-induced decoupling of TM and OHC's stereocilia. Because the endolymphatic overpressure would induce more significant both on the angle of the reticular lamina respect to the plane defined by the BM and in the RL-BM distance than in the position of the BM. There are several limitations of this study. First, because it was not a functional study, we couldn't check the important physiological changes such as OAE and auditory brainstem response during experiment. Second, the rate of infusion was greater than those of other studies. It might cause an exaggerated distortion of organ of Corti. With these points, this study is not thought as an appropriate animal model for Meniere's disease. However, It might have some meaning that it was the first observation about the change of microstructures of organ of Corti with increased pressure.

\section{REFERENCES}

1) Wit HP, Warmerdam TJ, Albers FW. Measurement of the mechanical compliance of the endolymphatic compartments in the guinea pig. Hear Res 2000;145(1-2):82-90.

2) Salt AN, Henson MM, Gewalt SL, Keating AW, DeMott JE, Henson OW Jr. Detection and quantification of endolymphatic hydrops in the guinea pig cochlea by magnetic resonance microscopy. Hear Res 1995;88(1-2):79-86.

3) Fleischer M, Schmidt R, Gummer AW. Compliance profiles derived from a three-dimensional finite-element model of the basilar membrane. J Acoust Soc Am 2010;127(5):2973-91.

4) Zagadou BF, Barbone PE, Mountain DC. Elastic properties of organ of Corti tissues from point-stiffness measurement and inverse analysis. J Biomech 2014;47(6):1270-7.

5) Tonndorf J. Endolymphatic hydrops: mechanical causes of hearing loss. Arch Otorhinolaryngol 1976;212(4):293-9.

6) Ruggero MA, Rich NC, Robles L, Shivapuja BG. Middle-ear response in the chinchilla and its relationship to mechanics at the base of the cochlea. J Acoust Soc Am 1990;87(4):1612-29.

7) Takeuchi S, Takeda T, Saito H. Pressure relationship between perilymph and endolymph associated with endolymphatic infusion. Ann Otol Rhinol Laryngol 1991;100(3):244-8.

8) Salt AN, Plontke SK. Endolymphatic hydrops: pathophysiology and experimental models. Otolaryngol Clin North Am 2010;43(5): 971-83.

9) Kakigi A, Salt AN, Takeda T. Effect of artificial endolymph injection into the cochlear duct on perilymph potassium. ORL J Otorhinolaryngol Relat Spec 2010;71 Suppl 1:16-8. 
10) Kakigi A, Takeda T. Effect of artificial endolymph injection into the cochlear duct on the endocochlear potential. Hear Res 1998;116(1-2): 113-8.

11) Brown DJ, Chihara Y, Curthoys IS, Wang Y, Bos M. Changes in cochlear function during acute endolymphatic hydrops development in guinea pigs. Hear Res 2013;296:96-106.

12) Ruding PR, Veldman JE, Berendsen W, Huizing EH. Scanning electron microscopy of hair cells, stereocilia and cross-linkage systems in experimentally induced endolymphatic hydrops. Eur Arch Otorhinolaryngol 1991;248(6):313-8.

13) Dunnebier EA, Segenhout JM, Dijk F, Albers FW. Sensory cell damage in two-phase endolymphatic hydrops: a morphologic evaluation of a new experimental model by low-voltage scanning techniques. Otol Neurotol 2001;22(5):655-61.

14) Yoon TH, Paparella MM, Schachern PA, Le CT. Cellular changes in Reissner's membrane in endolymphatic hydrops. Ann Otol Rhinol Laryngol 1991;100(4 Pt 1):288-93.

15) Rydmarker S, Horner KC. Morphological changes of hair cell stereocilia and tectorial membrane in guinea pigs with experimentally induced hydrops. Scanning Microsc 1990;4(3):705-13; discussion 713-4.

16) Zenner HP, Ruppersberg JP, Löwenheim H, Gummer AW. Pathophysiology: transduction and motor disturbances of hair cells by endolymphatic hydrops and transitory endolymph leakage. In: Harris JP, editor. Ménière's Disease. The Hague, The Netherlands: Kugler Publications;1999. p.203-17.

17) Zenner HP, Reuter G, Zimmermann U, Gitter AH, Fermin C, LePage EL. Transitory endolymph leakage induced hearing loss and tinnitus: depolarization, biphasic shortening and loss of electromotility of outer hair cells. Eur Arch Otorhinolaryngol 1994;251(3):143-53. 